\title{
Design and Synthesis of Thiadiazole Derivatives as Antidiabetic Agents
}

\author{
Prasanna A Datar* and Tejashree A Deokule \\ Department of Pharmaceutical Chemistry, Sinhgad Institute of Pharmacy, Narhe, Pune, India
}

\begin{abstract}
Thiadiazole compounds have versatile activities such as anti-microbial, anti-inflammatory, anticancer, etc. Present study involves design of thiadiazole compounds as antidiabetic agent using docking studies. The designed molecules were synthesized and subjected to antidiabetic activity by in vitro and in vivo method. Compound TD7 was found to show potent antidiabetic activity in alloxan induced diabetes rat model. Molecular docking revealed that synthesized derivatives and target proteins were actively involved in binding and had significant correlation with biological activity.
\end{abstract}

\section{Keywords: Diabetes; Thiadiazole; Docking; PPAR}

Abbreviations: CMC: Caboxy Methyl Cellulose; DNSA: Dinitro Salicylic Acid; FTIR: Fourier Transform Infra Red; IDDM: Insulin dependent diabetes mellitus; $\mathrm{Mg} / \mathrm{dl}$ : milligram per deciliter; NIDDM: Non insulin dependent diabetes mellitus; NMR: Nuclear Magnetic Resonance; P: Probability; PPA: Porcine Pancreatic $\alpha$-amylase; PDB: Protein Data Bank; PPAR: Peroxisome Proliferating Activator Enzyme; PPM: parts per million; RBF: Round Bottom Flask; SEM: Standard Error Mean; TLC: Thin Layer Chromatography; TMS: Tetramethylsilane; UV: Ultra-violet

\section{Introduction}

\section{Diabetes}

Diabetes is a group of diseases characterized by high blood glucose level. When a person has diabetes, the body either does not produce enough insulin or is unable to use its own insulin effectively. Glucose builds up in the blood and causes a condition that, if not controlled, can lead to serious health complications and even death. The risk of death for a person with diabetes is twice the risk of a person of similar age who does not have diabetes. Diabetes mellitus is a heterogeneous clinical disorder with numerous causes. Two main classifications of diabetes mellitus exist, idiopathic and secondary. Idiopathic diabetes is divided into two main types: Insulin dependent and Non-insulin dependent. Non insulin dependent diabetes mellitus (NIDDM or type 2 diabetes) is characterized by milder hyperglycemia and rarely leads to ketoacidosis. It leads to nephropathy, neuropathy, and retinopathy.

Diabetes mellitus, long considered a disease of minor significance to world health, is now taking its place as one of the main threats to human health in the $21^{\text {st }}$ century. The incidence of the disease currently is estimated to reach 210 million by the year 2010 and 300 million by the year 2025 [1]. Most cases will be of type 2 diabetes, which is strongly associated with a sedentary life style and obesity. Nowadays diabetes is becoming a leading cause of death in most nations. 1,3,4-thiadiazole and its derivatives possesses wide range of therapeutic activities like activities like antimicrobial [2], antifungal, diuretics, antiulcer [3], antimycobacterial [4], antioxidant/radio-protective [5], anti-inflammatory, anticonvulsant, antidepressant, anticancer, anti-leshmanial [6-7], carbonic anhydrase inhibitors [8] and antidiabetic [9]. Due to presently known limitations of liver toxicity in the use of thiazolidinediones, it is necessary to find new heterocyclic compounds with equivalent potency as that of thiazolidinedione. Thiadiazole compounds are known to bind peroxisome proliferator activating receptor (PPAR) effectively [10]. Therefore present study involves design of thiadiazole compounds on the basis of docking studies on peroxisome proliferator activating receptor (PPAR) effectively and to find important binding residues. The knowledge of binding residues would help in design of novel thiadiazole molecules as antidiabetic agents.

\section{Chemistry}

Designing of new molecules was done with help of docking. Virtual screening has emerged as a reliable, cost-effective and time-saving technique for the discovery of lead compounds. The virtual screening approach for docking small molecules into a known protein structure is a powerful tool for drug design that has become an integral part of the drug discovery process in recent years. Theoretically the application of virtual screening is constrained only by the chemical compounds features that can be calculated and the relation between these features and the targeted protein can be established. Practical implementation of virtual screening application requires some considerations. If the site geometry is not known, as is often the case, the design should be based on other ligand molecules that bind well to the site. If the molecules are rigid, the problem becomes one of identifying the substructures or active groups that contribute to the fit. If the site geometry is known, molecules with good affinity for the site can be built by joining the groups with alternative molecular scaffolding. In present study, thiadiazole scaffold was considered as replacement to thiazolidinedione while considering its expected interaction with PPARgamma receptor as per the previous literature reports [9]. Thiazolidinediones belongs to a chemical class that has a different pharmacological action than the sulfonylureas, biguanides, or the (alpha)-glucosidase inhibitors. Synthesized thiadiazole compounds were tested for alpha-glucosidase inhibition to verify whether these compounds work by mechanism similar to thiazolidinediones or else.

In vivo study was carried out on alloxan induced diabetes rat model for all the synthesized molecules using glibenclamide, a second generation sulphonylurea as standard since it was used as a reference standard compound in the study of reference analogous compounds $[11,12]$. Molecular docking studies were performed by using Glide v5.6 (Schrodinger, LLC) [13]. The X-ray crystal of peroxisome proliferator-activated receptor gamma (PPARgamma) in complex with rosiglitazone (PDB id. 1FM6) was obtained from the RCSB protein Data Bank (PDB) and utilized in order to get the detailed insights of ligand-protein structure in this study.

*Corresponding author: Prasanna A Datar, Department of Pharmaceutical Chemistry, Sinhgad Institute of Pharmacy, Narhe, Pune, India, Tel: +91 9823161187 E-mail: d_pras_anna@rediffmail.com

Received March 26, 2014; Accepted April 26, 2014; Published April 28, 2014

Citation: Datar PA, Deokule TA (2014) Design and Synthesis of Thiadiazole Derivatives as Antidiabetic Agents. Med chem 4: 390-399. doi:10.4172/21610444.1000170

Copyright: @ 2014 Datar PA, et al. This is an open-access article distributed under the terms of the Creative Commons Attribution License, which permits unrestricted use, distribution, and reproduction in any medium, provided the original author and source are credited. 
Citation: Datar PA, Deokule TA (2014) Design and Synthesis of Thiadiazole Derivatives as Antidiabetic Agents. Med chem 4: $390-399$. doi:10.4172/2161-0444.1000170

Protein structure was added with hydrogens and then only hydrogens were energetically optimized. In case of docking poses showing high score, some manual adjustment were done to remove large steric hindrance and final complex structures were subjected to energy minimization using OPLS force field in Schrodinger software. During the energy minimization procedure firstly whole protein structure was fixed and secondly that amino acid residues within $3.5 \AA$ from the each ligand were relaxed.
Rosiglitazone was removed from the complex and the reported thiadiazole compounds were docked in PPARgamma protein and their binding interaction was validated with previously reported binding residues. These binding residues were used as reference guide. Several thiadiazole compounds have been reported for their antidiabetic effect by acting as agonist at PPAR gamma [11,12]. Docking was performed selectively on reported thiadiazole analogues Tables 1 and 2 for which antidiabetic activity is known to be potent and comparable to standard.

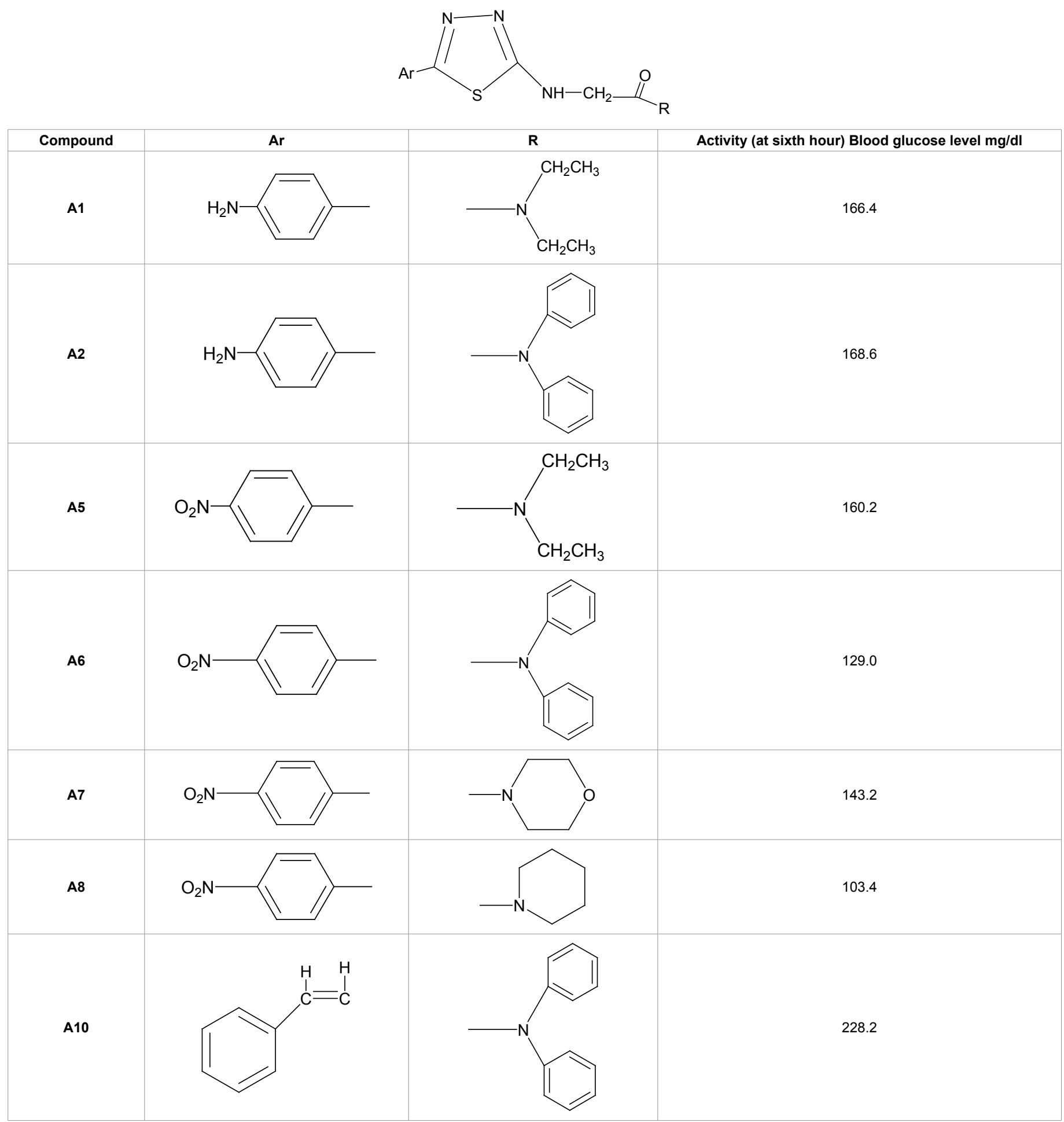

Table 1: Thiadiazole compounds $\mathrm{A} 1$ to $\mathrm{A} 10$ as antidiabetic were used as reference compounds. 
Citation: Datar PA, Deokule TA (2014) Design and Synthesis of Thiadiazole Derivatives as Antidiabetic Agents. Med chem 4: $390-399$. doi:10.4172/2161-0444.1000170

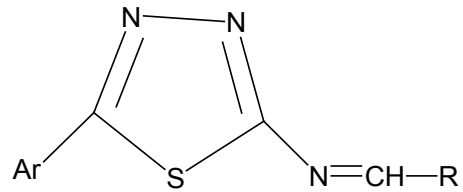

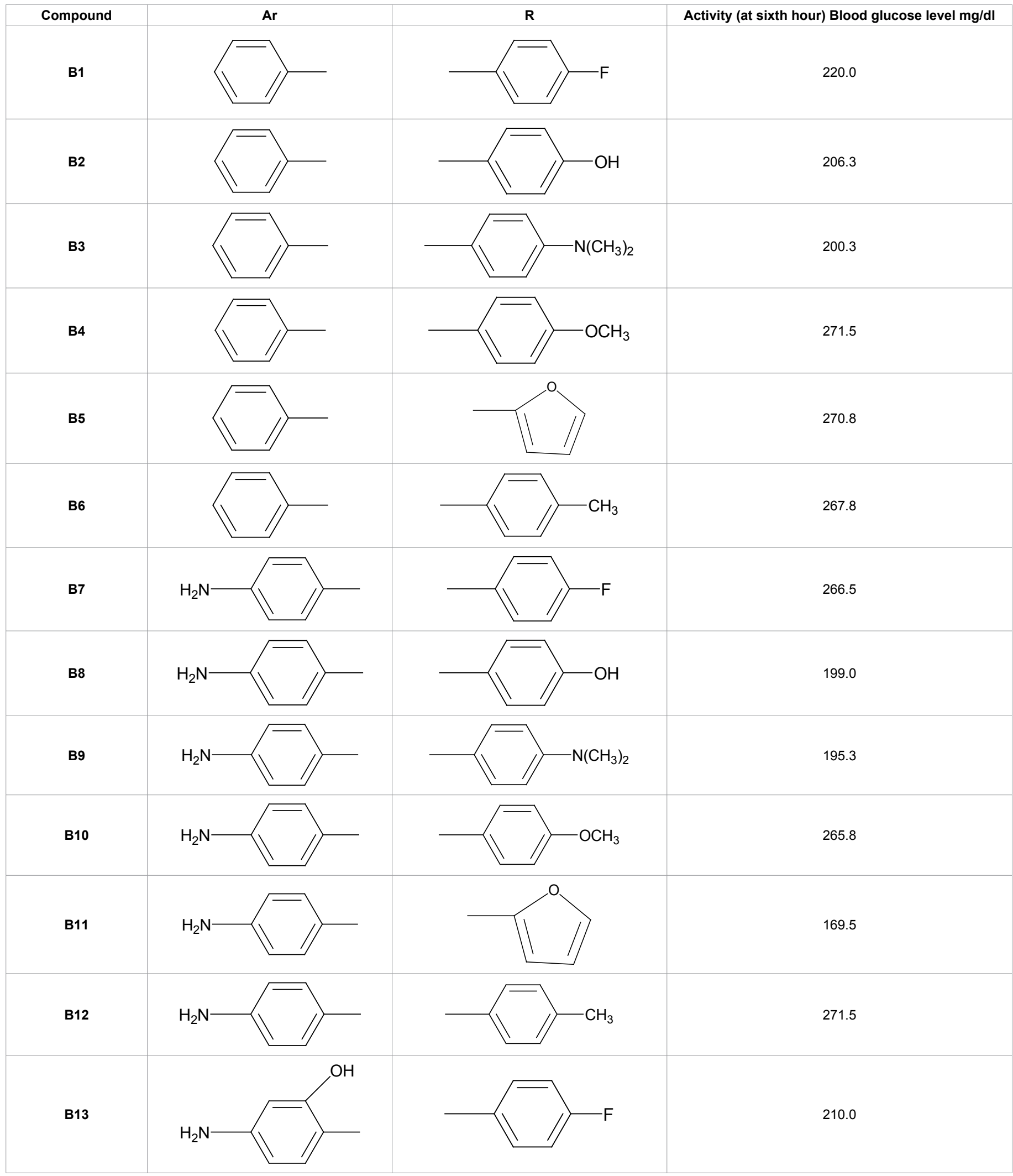


Citation: Datar PA, Deokule TA (2014) Design and Synthesis of Thiadiazole Derivatives as Antidiabetic Agents. Med chem 4: $390-399$. doi:10.4172/2161-0444.1000170

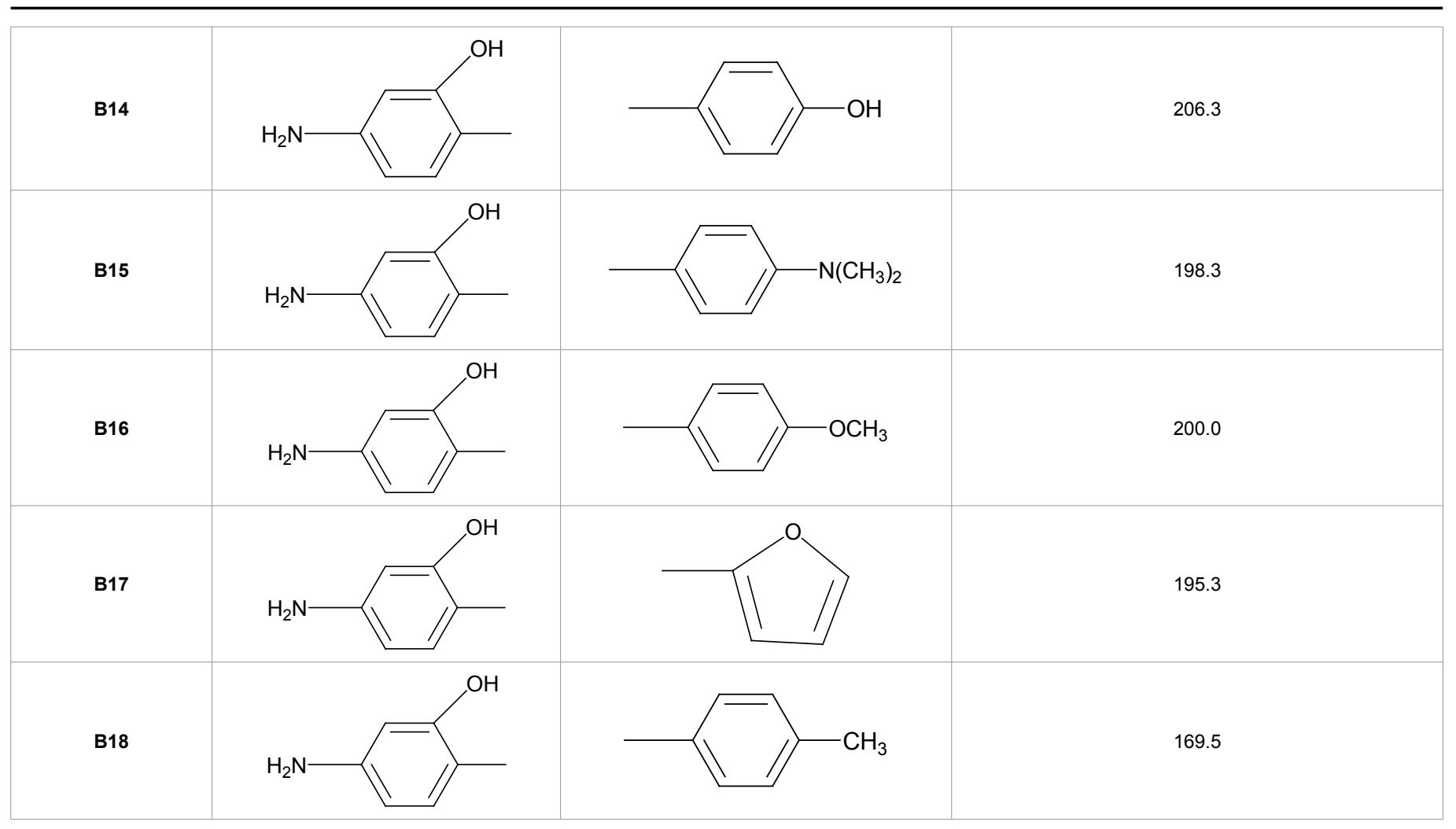

Table 2: Thiadiazole compounds B1 to B18 as antidiabetic were used as reference structures.

This study helped in understanding possible orientations and conformation of thiadiazole compounds and their resulting position and binding interaction in the pocket. Similar to the thiazolidinedione group of rosiglitazone, the carboxyl group of reference compound was found to show hydrogen bonds with Tyr473 as well as Ser289 and His449.

\section{Gscore}

The docking studies were performed using standard precision mode of Glide. The results of the docking studies were generated in the form of Gscore. The more negative value of Gscore means, the compound is more potent and indicates good binding potential. Gscore in Glide has components such as steric, electrostatic, hydrophobic, charge-charge, charge-polar, H-bonding and metal-ligand interactions. Besides at the expense of penalties of freezing rotatable bonds, buried polar bonds and desolvation interactions are also considered after post-docking minimization. Apart from Gscore, other parameters like energy and Emodel were also taken into consideration for the evaluation of the docking results. Emodel is composed of energy of grid score, the binding affinity of the ligand and the internal strain energy. The values of energy and Emodel were found to be significantly closer to the values of the standard rosiglitazone.

\section{$\mathrm{H}$-Bond interaction}

As $\mathrm{H}$-bond is an influential parameter, which governs binding affinity of the drug compounds, this parameter was also taken into consideration. The number of $\mathrm{H}$-bond interaction in the standard compounds rosiglitazone was compared with those of the designed thiadiazoles.

\section{Experimental Methods}

The starting materials were characterized with the help of melting point in open capillaries using melting point apparatus (VEEGO VMP-D) and are uncorrected. Progress of the reaction and purity of the compounds was confirmed by TLC plates by using appropriate mobile phase and spots were rendered visible by exposing to UV light and iodine fumes. FTIR spectra were recorded by JASCO FTIR 4100 series in the range of $4000-400 \mathrm{~cm}^{-1} .{ }^{1} \mathrm{HNMR}$ spectra were recorded by Varian-NMR-Mercury 300 using DMSO as a solvent and their chemical shifts are recorded in $\delta$ parts per million (ppm) units with respect to tetramethylsilane (TMS) as an internal standard.

\section{Scheme of synthesis}

Figure 1 shows scheme 1 [11] of synthesis for compound TD1 to TD6. Figure 2 shows scheme 2 for synthesis of compound TD7 [12].

\section{General Procedure}

The compounds TD1-TD6 were synthesized using similar procedure [14]. The yield and m.p. of synthesized compounds are listed in Table 6.

\section{Step 1: (Scheme 1 and 2) Synthesis of 2-amino-5-aryl-1,3,4- thiadiazole}

Thiosemicarbazide (0.01 mole), aryl carboxylic acid ( 0.01 mole), and conc. Sulphuric acid $(5 \mathrm{ml})$ were refluxed for 2 hours. The reflux was continued for further time of span until we get a distinct single point on TLC. This hot mixture was poured onto crushed ice. The solid separated was filtered out and washed with water. It was recrystallized from ethanol to give compound of step 1. This step is common for compounds TD1-TD7

Step 2: (Scheme 1): Synthesis of 5-aryl-1,3,4-thiadiazol-2-yl) acetamide (1 -6)

Product of step 1(5-aryl-1,3,4-thiadiazol-2-amine) (0.01 mole) and acetyl chloride $(0.01$ mole) were taken in RBF. The mixture was irradiated in a microwave oven for 3 mins with $40 \%$ power, at intervals of 30s. After the completion of reaction, ice cold water was added to 


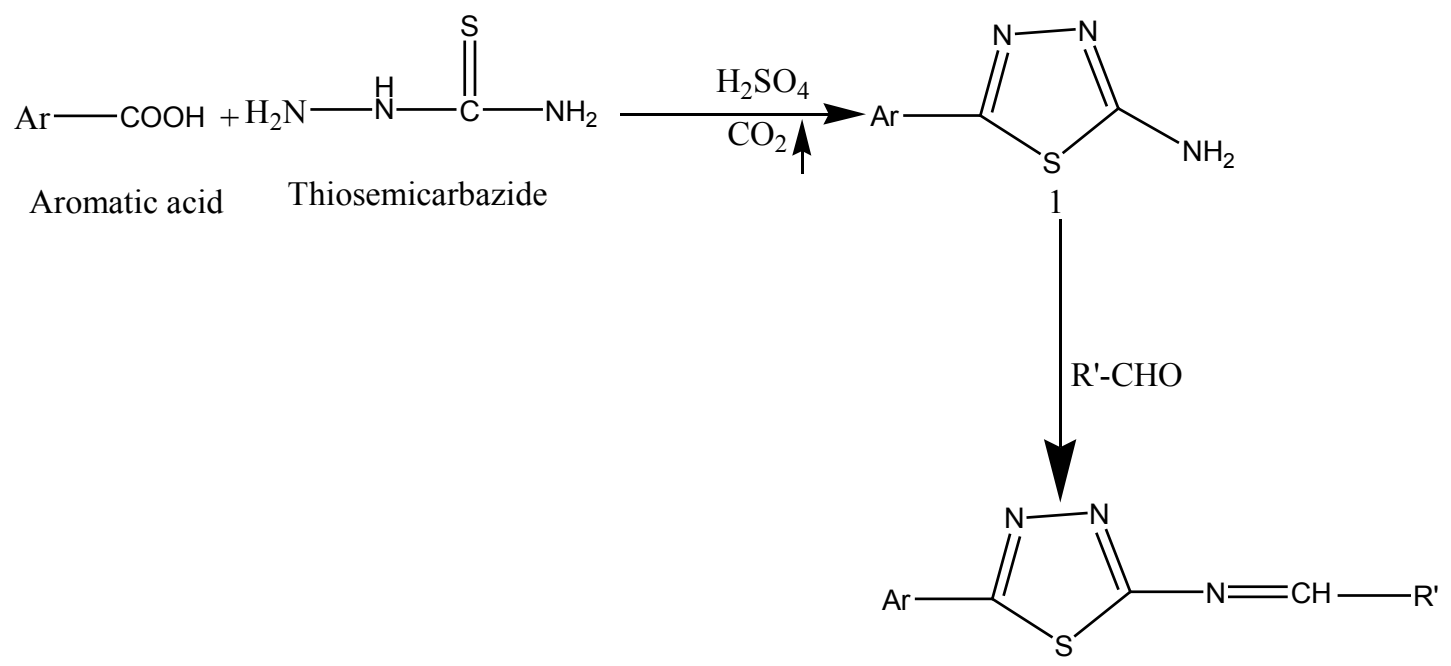

Figure 1: Scheme of synthesis for TD1- TD6.<smiles>NNC(=S)NN</smiles>

Aromatic acid Thiosemicarbazide<smiles>[R20]C(C)(C)[14C](=O)O[Na]</smiles>

Reflux<smiles>O=C(Cl)C[Hg]C1CCCC1</smiles><smiles></smiles><smiles>Ic1ccccc1</smiles><smiles>[R]C(=O)CNc1nnc([Al])s1</smiles>

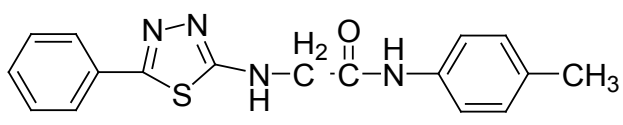

Compound TD7

Figure 2: Scheme of synthesis for TD7.

the reaction mixture and solid thus separated was filtered and dried to get 5-aryl-1,3,4-thiadiazol-2-yl) acetamide, and recrystallized from ethanol.

Step 2: (Scheme 2): For synthesis of 2-(5-phenyl-1,3,4thiadiazol-2-ylamino)-N-p-tolylacetamide

Compounds from Step 1 (0.5 mol) was dissolved in glacial acetic acid $(25 \mathrm{ml})$ containing $25 \mathrm{ml}$ of saturated solution of sodium acetate. In case the substance did not dissolve completely, the mixture was warmed and the solution was cooled in ice bath with stirring. To this chloroacetyl chloride was added dropwise $(0.06 \mathrm{~mol})$ so that, vigorous reaction did not take place. After half an hour white product was separated and filtered. The product was washed with $50 \%$ aqueous acetic acid and finally with water. It was purified by recrystallization from absolute alcohol. 


\section{Step 3: (Scheme 2): For synthesis of 2-(5-phenyl-1,3,4-} thiadiazol-2-ylamino)-N-p-tolylacetamide (TD7)

A mixture of product from step $2(0.01 \mathrm{~mol})$ was taken in $25 \mathrm{ml}$ of absolute alcohol and $0.01 \mathrm{~mol}$ of aryl derivative was added and refluxed for $4 \mathrm{hr}$. It was purified by recrystallization from aqueous alcohol.

\section{Pharmacology}

\section{In vitro activity}

Porcine Pancreatic $\alpha$-amylase (PPA) was used for preliminary screening of $\alpha$-amylase inhibitors from the compounds. The inhibition assay was performed using the chromogenic Dinitro Salicylic Acid (DNSA) method [15]. The total assay mixture composed of $500 \mu \mathrm{L}$ of $0.02 \mathrm{M}$ sodium phosphate buffer ( $\mathrm{pH} 6.9$ containing $6 \mathrm{mM}$ sodium chloride), 0.04 units of PPA solution, and compounds at concentration from $0.1-1.5 \mathrm{mg} / \mathrm{ml}(\mathrm{w} / \mathrm{v})$ were incubated at $37^{\circ} \mathrm{C}$ for $10 \mathrm{~min}$. After pre-incubation, $500 \mu \mathrm{l}$ of $1 \%(\mathrm{v} / \mathrm{v})$ starch solution in the above buffer was added to each tube and incubated at $37^{\circ} \mathrm{C}$ for $15 \mathrm{~min}$. The reaction was terminated with $1.0 \mathrm{ml}$ DNSA reagent, placed in boiling water bath for $5 \mathrm{~min}$, cooled to room temperature, diluted, and the absorbance measured at $540 \mathrm{~nm}$. The control reaction representing 100\% enzyme activity did not contain any compound. To eliminate the absorbance produced by compound, appropriate compound controls were also included. One unit of enzyme activity is defined as the amount of enzyme required to release one micromole of maltose from starch per min under the assay conditions.

For the determination of the inhibitor concentration at which 50 $\%$ inhibition of enzyme activity occurs $\left(\mathrm{IC}_{50}\right)$, the assay was performed as above except that the compound concentrations were varied from 0.1-150 $\mu \mathrm{g}$. Acarbose was used as a positive control at a concentration range of $6.5 \mu \mathrm{g}-32.8 \mu \mathrm{g}$. The $\mathrm{IC}_{50}$ values were determined from plots of percent inhibition versus log inhibitor concentration and calculated by logarithmic regression analysis from the mean inhibitory values. The $\mathrm{IC}_{50}$ values were defined as the concentration of the compound, containing the $\alpha$-amylase inhibitor that inhibited $50 \%$ of the PPA activity. The results of in vitro study are presented in Table 7 .

\section{In vivo activity}

Alloxan induced diabetes model [16] The acclimatized animals were kept fasting for $24 \mathrm{hrs}$ with water and alloxan monohydrate (90 $\mathrm{mg} / \mathrm{kg}$ i.p.) in normal saline was administered. After $1 \mathrm{hr}$ of alloxan administration the animals were given water. The $5 \%$ dextrose solution was given in feeding bottle for a day to overcome the early hypoglycemic phase. The blood glucose was monitored by Retro orbital flexus method [17]. The blood was dropped on the dextrostrip reagent pad. The strip was inserted in one touch simple select glucometer and readings were noted. After $4^{\text {th }}$ day, rats having blood glucose level beyond $200 \mathrm{mg} / \mathrm{dl}$ of blood were selected for the study and divided in 6 groups $(n=6)$. The quantity of $1,3,4$-thiadiazole derivatives equivalent to average human intake $200 \mathrm{mg} / \mathrm{kg}$ at a time was calculated for single dose $35 \mathrm{mg} / \mathrm{kg}$. The test compounds were administered orally by mixing with CMC (1\%) solution. The blood glucose levels were monitored at different time 0 , $1,3,6 \mathrm{hr}$ respectively. Glibenclamide was used as standard $10 \mathrm{mg} / \mathrm{kg}$. The results of in vivo study are presented in Table 8 .

\section{Results and Discussion}

\section{Docking of thiadiazole compounds}

Molecular docking studies were performed in our laboratory by using Glide v5.6 (Schrodinger, LLC). PDB 1FM6 containing PPARgamma bound to rosiglitazone was selected for docking. Docking of literature based reported antidiabetic molecules were performed to know insights of interaction of thiadiazole compounds with the active site residues. The information of important amino acid residues was compared to new designed molecules. The choice of best-docked structure for each ligand is made using a model energy score (Emodel) that combines the energy grid score, the binding affinity predicted by GlideScore, lipophilicity, hydrogen bond, internal energy, van der waals energy. Newly designed molecules that have comparable docking score values to literature based reported docked molecules were selected for synthesis.

Docking studies were performed on reported thiadiazole series. Docking studies revealed that the reported molecules had typical interactions. This study shows thiadiazole has different binding mode as compared to binding interactions of rosiglitazone in crystal structure 1FM6.

Table 1 and 2 shows thiadiazole compounds series A1 to A10 [11] and $\mathrm{B} 1$ to $\mathrm{B} 18$ [12] as reference antidiabetic compounds obtained from the literature. Antidiabetic activity has been reported for these series of compounds on alloxan induced rat model. The activity has been reported in terms of blood glucose level in $\mathrm{mg} / \mathrm{dl}$ after the period of sixth hour. The reported antidiabetic activity values showed that compounds $\mathrm{A} 6$ and $\mathrm{A} 8$ have higher activity than $\mathrm{B} 11$ and $\mathrm{B} 18$ compounds.

Table 3 shows results of docking study of reported thiadiazole series, which was used as guideline for the study of newly designed thiadiazole compounds. Table 3 shows the hydrogen bonding distances of the reported thiadiazole series of compounds. The residues obtained from these studies were compared with the previous reported docking study of thiadiazole compounds $[9,10]$. The compounds were ranked as per the docking score. This docking study reveals the importance of amino acid residues found to have significant interactions. From this study it was observed that compounds that have lowest hydrogen bonding distances with Ser342 are also higher active amongst the selected series of thiadiazole compounds (Figure 3 ).

\section{Docking}

In Table 3, compounds A2, A5, A6, A8 and B2, B10, B15, B16, B18 were found to have meaningful hydrogen bonding interaction with the amino acid residue Ser342. It can be observed in Table 3, that distance between amino acid residue Ser342 and ligands such as B3, B4, B13, $\mathrm{B} 10, \mathrm{~B} 12$ and $\mathrm{A} 10$ were larger than $3.5 \AA$ indicating weak binding interactions. Distances larger than $3.5 \AA$ were considered as weak interactions for attraction between hydogen bond donor and hydrogen bond acceptor. The lower activity of these compounds may be due to weaker binding interactions.

Table 4 consists of first seven virtually designed molecules that were selected based on no steric clash and lower GScore values.

Table 5 shows Gscore and H-bonding distances for designed compounds TD1 to TD7. TD1 was found to have hydrogen boning with Cys285 in which sulfer H-bonds with nitrogen of thiadiazole ring system. TD2, TD3, TD5 and TD7 forms H-bond with Ser342. TD4 shifts away from Ser342 and forms H-bond with Arg288. The molecule TD7 forms H-bonding with Ser342 and bends towards ILE-281. The bending is due to absence of double bond linkage in the molecule TD7. Such interaction is not possible in TD1 to TD6. Electron rich substitutions on Ar position shifts the molecule towards other side of the cavity i.e. towards Arg288 residue. TD4 has no H-bonding with Ser342 and drifts towards Arg288. R group substitutions that vary from acetyl or methyl group of toluidine showed no steric clashes with Ile281. Molecules TD3, TD4 and TD5 drift away from Ile281 at their R end position. Molecule TD6 did not have H-bonding with Ser342 but it is closer to Ile281 at its R end position (Figure 4). 
Citation: Datar PA, Deokule TA (2014) Design and Synthesis of Thiadiazole Derivatives as Antidiabetic Agents. Med chem 4: $390-399$. doi:10.4172/2161-0444.1000170

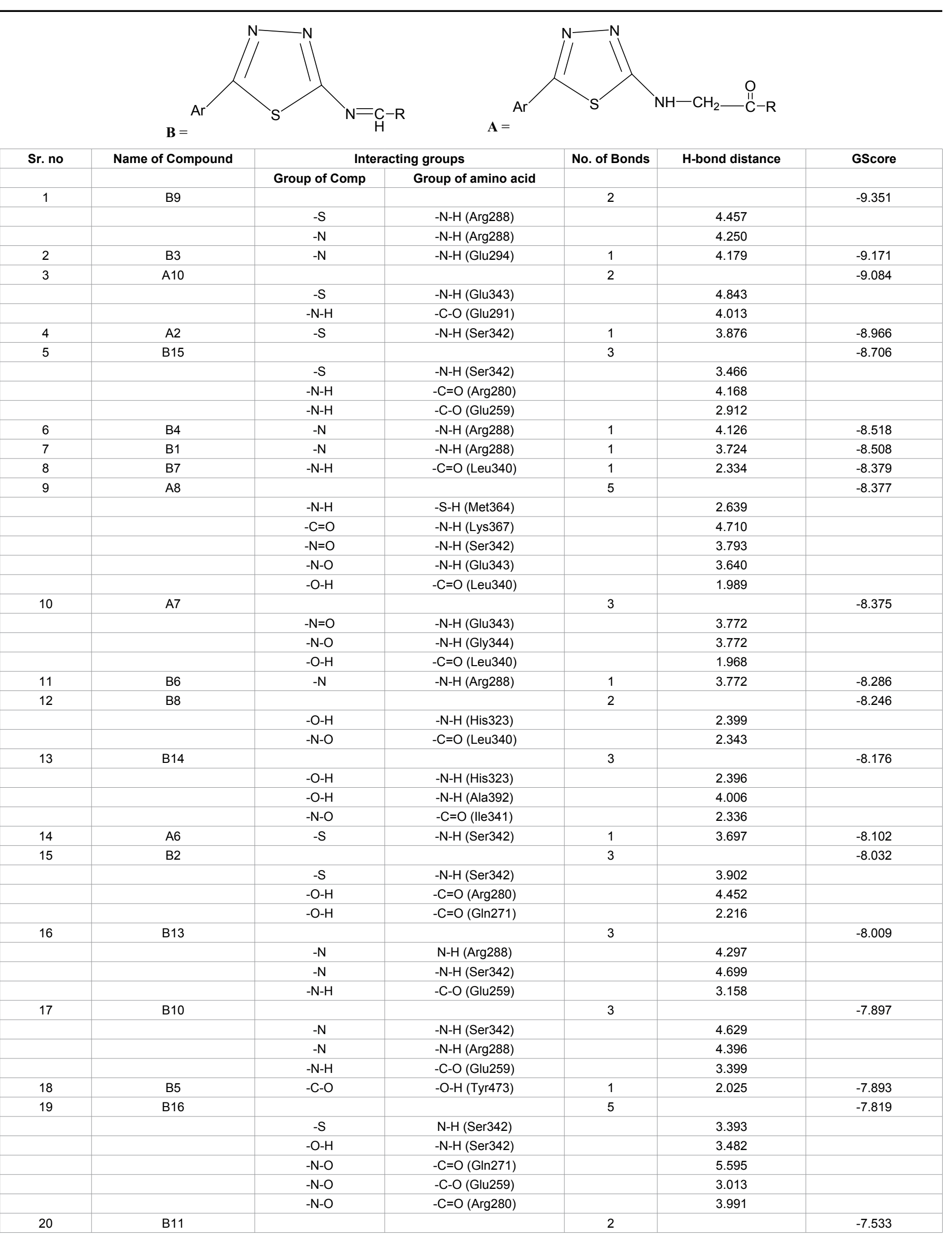


Citation: Datar PA, Deokule TA (2014) Design and Synthesis of Thiadiazole Derivatives as Antidiabetic Agents. Med chem 4: $390-399$. doi:10.4172/2161-0444.1000170

\begin{tabular}{|c|c|c|c|c|c|c|}
\hline & & $-\mathrm{N}-\mathrm{O}$ & $-\mathrm{C}=\mathrm{O}$ (Val339) & & 4.401 & \\
\hline & & $-\mathrm{C}-\mathrm{O}$ & -O-H (Tyr473) & & 2.084 & \\
\hline \multirow[t]{4}{*}{21} & B12 & & & 3 & & -7.495 \\
\hline & & $-\mathrm{N}$ & $-\mathrm{N}-\mathrm{H}(\mathrm{Arg} 288)$ & & 4.587 & \\
\hline & & $-\mathrm{N}$ & $-\mathrm{N}-\mathrm{H}(\mathrm{Ser} 342)$ & & 4.663 & \\
\hline & & $-\mathrm{N}-\mathrm{O}$ & -C-O (Glu259) & & 3.858 & \\
\hline \multirow[t]{4}{*}{22} & B18 & & & 3 & & -7.444 \\
\hline & & $-\mathrm{N}$ & -N-H (Ser342) & & 2.816 & \\
\hline & & $-\mathrm{O}-\mathrm{H}$ & $-\mathrm{N}-\mathrm{H}$ (Glu343) & & 4.084 & \\
\hline & & $-\mathrm{O}-\mathrm{H}$ & $-\mathrm{N}-\mathrm{H}(\mathrm{Arg} 288)$ & & 4.417 & \\
\hline \multirow[t]{4}{*}{23} & B17 & & & 3 & & -6.898 \\
\hline & & $-\mathrm{N}-\mathrm{H}$ & -C-O (Glu259) & & 2.537 & \\
\hline & & $-\mathrm{N}$ & $-\mathrm{N}-\mathrm{H}(\mathrm{Ser} 342)$ & & 4.658 & \\
\hline & & $-\mathrm{N}$ & $-\mathrm{N}-\mathrm{H}(\operatorname{Arg} 288)$ & & 4.620 & \\
\hline \multirow[t]{3}{*}{24} & A1 & & & 2 & & -6.841 \\
\hline & & $-\mathrm{N}$ & $-\mathrm{N}-\mathrm{H}(\mathrm{Ser} 342)$ & & 4.020 & \\
\hline & & $-\mathrm{N}-\mathrm{O}$ & -C-O (Glu259) & & 2.557 & \\
\hline \multirow[t]{3}{*}{25} & A5 & & & 2 & & -6.677 \\
\hline & & $-\mathrm{N}$ & $-\mathrm{N}-\mathrm{H}(\mathrm{Ser} 342)$ & & 3.298 & \\
\hline & & $-\mathrm{O}-\mathrm{H}$ & -C-O (Glu259) & & 2.178 & \\
\hline
\end{tabular}

Table 3: Hydrogen bonding distances for reported thiadiazole series of compounds.

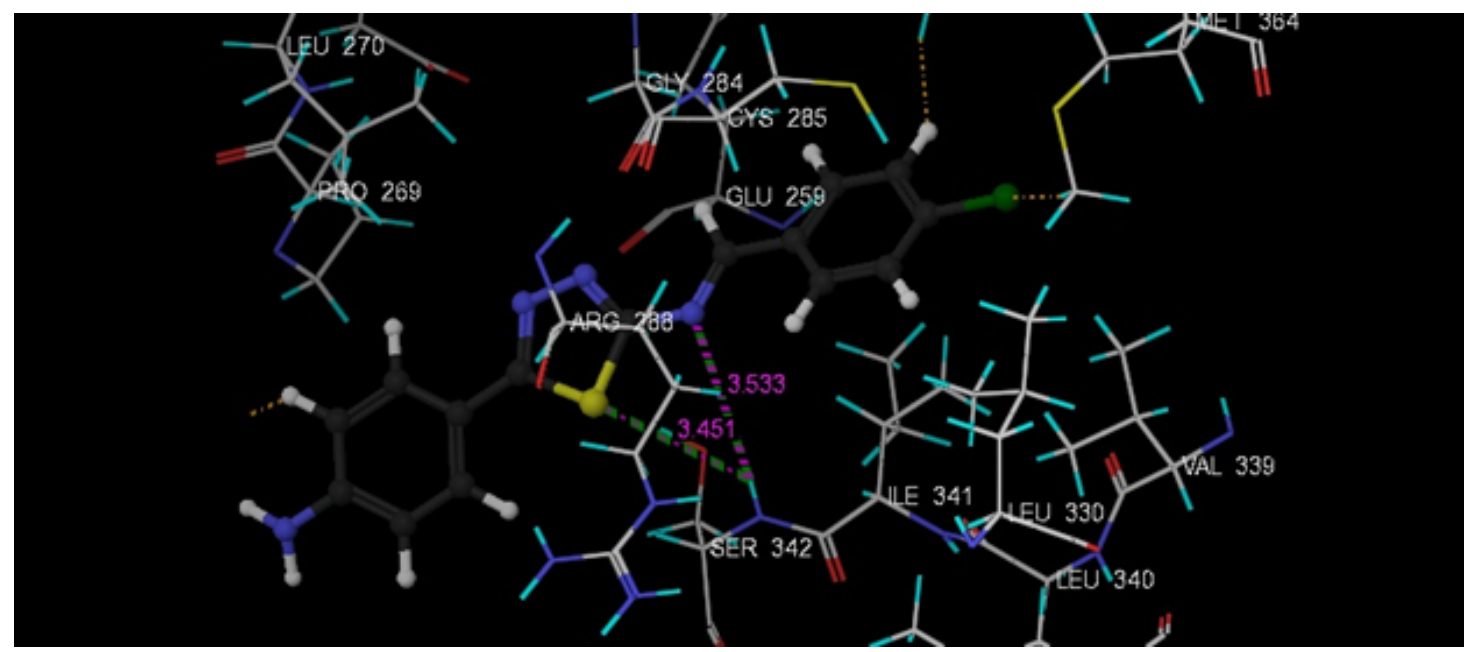

Figure 3: Docking of TD3 showing H-bonding with Ser342 and steric clash of Chlorine group at R position with Met364.

\section{Spectral Data of synthesized compounds}

Table 6 gives the characterization data of the synthesized compounds.

Compound TD1: N-(5-(4-nitrophenyl)-1,3,4-thiadiazol-2-yl) acetamide: IR ( $\mathrm{KBr})$ : 3119 (Ar C-H str), 1606 (C=O str), $1524\left(\mathrm{NO}_{2}\right)$, 632 (C-S str), 1283 (C-N str): ${ }^{1} \mathrm{H}$ NMR (DMSO): $\delta$ 8.2-8.3 (S, Ar. CH), $1.4\left(\mathrm{~S}, \mathrm{CH}_{3}\right), 4.4(\mathrm{~m},-\mathrm{NH})$.

Compound TD2: N-(4-nitrobenzilidene)-5-phenyl-1,3,4thiadiazol-2-amine: IR (KBr): $3106(\mathrm{Ar} \mathrm{C}-\mathrm{H}), 1531\left(\mathrm{NO}_{2}\right), 1345(\mathrm{C}-\mathrm{N}$ str), 626 (C-S str): ${ }^{1} \mathrm{H}$ NMR (DMSO): $\delta 8-9$ (S, Ar. CH), 10 (S, -CH).

Compound TD3: N-(4-chlorobenzylidene)-5-(4-aminophenyl)1,3,4thiadiazol-2-amine: IR (KBr): 3086 (Ar C-H), 3328 (-NH), 1309 (C-N), 608 (C-S), 590 (Cl): ${ }^{1} \mathrm{H}$ NMR (DMSO): $\delta 4\left(\mathrm{NH}_{2}\right), 8.1(\mathrm{~N}=\mathrm{C}-\mathrm{H})$, 6.5-7.5 (Ar C-H),

Compound TD4: N-(5-phenyl-1,3,4-thiadiazol-2-yl) acetamide: IR (KBr): 1693 (Amide C=O), 3426 (Ar C-H), 1293 (C-N), 634 (C-S): ${ }^{1} \mathrm{H}$ NMR (DMSO): $\delta$ 7-8 (Ar- C-H), $2.4\left(-\mathrm{CH}_{3}\right)$.
Compound TD5: N-(4-chlorobenzylidene)-5-phenyl-1,3,4thiadiazol-2-amine: IR ( $\mathrm{KBr}): 1289(\mathrm{C}=\mathrm{N}$ in ring), $1689(\mathrm{C}-\mathrm{N}), 708$ $(\mathrm{C}=\mathrm{Cl}), 3071$ (Ar C-H), 615 (C-S): ${ }^{1} \mathrm{H}$ NMR (DMSO): $\delta$ 8.0-8.1 (S, -CH), 7.2-7.6 (Ar- C-H).

Compound TD6: N-(4-fluorobenzylidene)-5-(4-aminophenyl)1,3,4-thiadiazol-2-amine: IR (KBr): 3072 (Ar C-H), 3329 (N-H), 677 (C-S), 1626 (C-N), 3081.69 (C-H): ${ }^{1} \mathrm{H}$ NMR (DMSO): $\delta$ 7.2-7.6 (Ar C-H), $8.1(\mathrm{~N}=\mathrm{C}-\mathrm{H})$.

Compound TD7: 2-(5-phenyl-1,3,4-thiadiazol-2-ylamino)-N-ptolylacetamide: IR (KBr): 3126 (Ar C-H), 1302 (C-N), 616 (C-S), 1616 $(\mathrm{C}=\mathrm{O}):{ }^{1} \mathrm{H}$ NMR (DMSO): $\delta 7.2$ (Ar- C-H), $1.5\left(-\mathrm{CH}_{3}\right), 4$ (Ar C-NH).

\section{In vitro activity}

In vitro studies carried out on porcine alpha amylase showed that out of all molecules TD1 was found most potent. Acarbose, alphaglucosidase inhibitor was used as standard in vitro study. Acarbose lowers the rate of glucose absorption through delayed carbohydrate digestion and extended digestion time. It has been determined that acarbose may have the capability to permanently or temporarily stop 
Citation: Datar PA, Deokule TA (2014) Design and Synthesis of Thiadiazole Derivatives as Antidiabetic Agents. Med chem 4: $390-399$. doi:10.4172/2161-0444.1000170

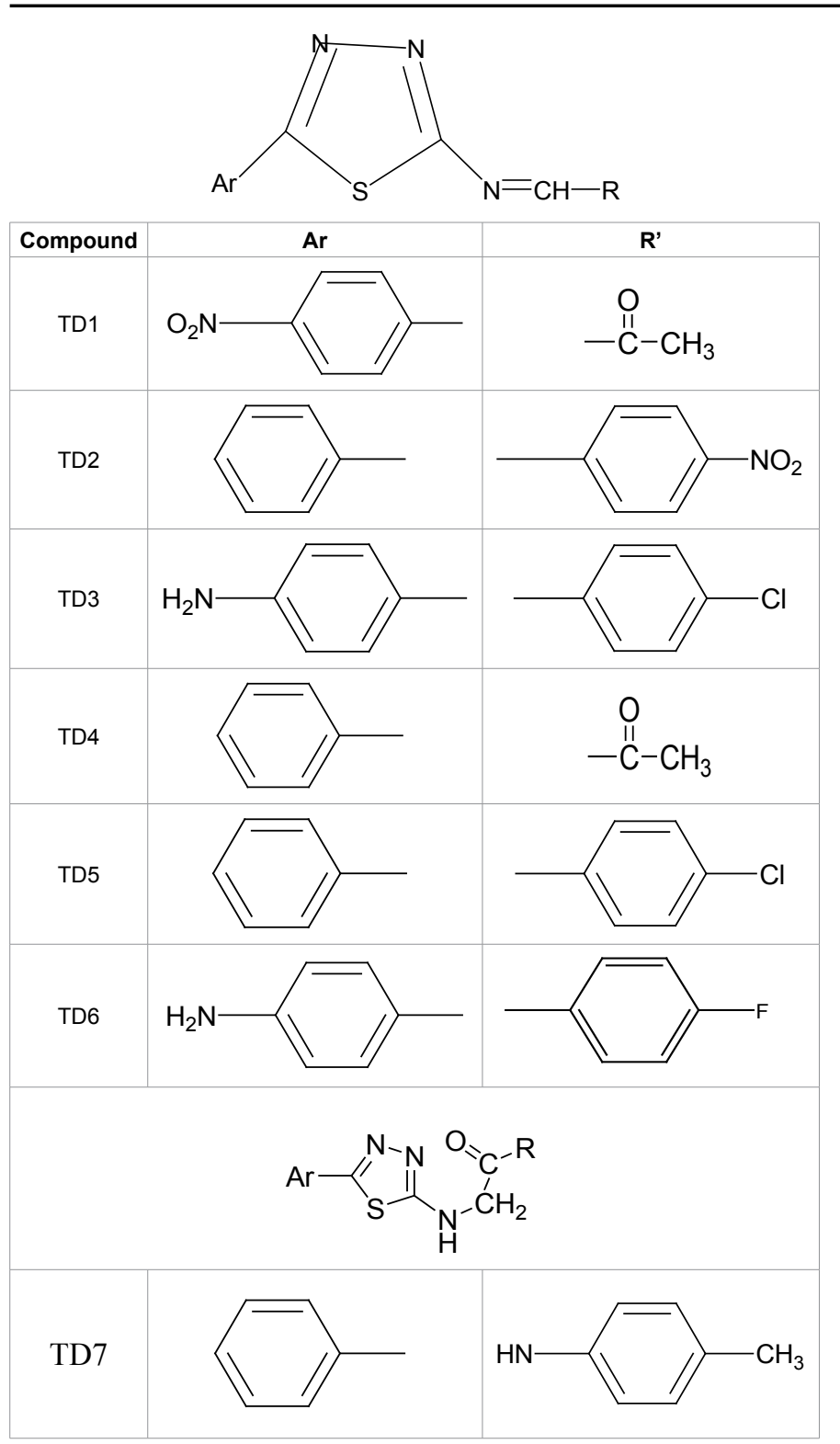

\begin{tabular}{|c|c|c|c|}
\hline Compound & Gscore & Interaction & H-bond distance \\
\hline TD1 & -8.023 & Cys285 & 2.480 \\
\hline TD2 & -7.404 & Ser342 & 2.527 \\
\hline TD3 & -8.895 & Ser342 & 3.183 \\
\hline TD4 & -8.422 & Arg288 & 3.451 \\
\hline TD5 & -8.108 & Ser342 & 2.609 \\
\hline TD6 & -8.115 & - & 2.597 \\
\hline TD7 & -8.392 & Ser342 & - \\
\hline $\begin{array}{c}\text { Rosiglitazone } \\
\text { (Standard) }\end{array}$ & -9.94 & Glu343 & 2.206 \\
\hline
\end{tabular}

Table 5: Gscore and Hydrogen bonding distances for the designed molecules.

\begin{tabular}{|c|c|c|c|c|c|}
\hline Compound & Mol. Formula & Mol Wt. & $\begin{array}{c}\text { M.P. } \\
\text { (uncorrected) }\end{array}$ & Rf value & \% Yield \\
\hline TD1 & $\mathrm{C}_{10} \mathrm{H}_{8} \mathrm{~N}_{4} \mathrm{O}_{3} \mathrm{~S}$ & 264.26 & $58-60$ & 0.76 & 71.97 \\
\hline TD2 & $\mathrm{C}_{15} \mathrm{H}_{10} \mathrm{~N}_{4} \mathrm{O}_{2} \mathrm{~S}$ & 310.05 & $108-110$ & 0.68 & 39.83 \\
\hline TD3 & $\mathrm{C}_{15} \mathrm{H}_{11} \mathrm{~N}_{4} \mathrm{SCl}$ & 317.89 & $198-202$ & 0.84 & 57.87 \\
\hline TD4 & $\mathrm{C}_{10} \mathrm{H}_{9} \mathrm{~N}_{3} \mathrm{SO}$ & 219.6 & $110-112$ & 0.64 & 77.89 \\
\hline TD5 & $\mathrm{C}_{15} \mathrm{H}_{10} \mathrm{~N}_{3} \mathrm{SCl}$ & 299.78 & $118-120$ & 0.77 & 32.44 \\
\hline TD6 & $\mathrm{C}_{15} \mathrm{H}_{11} \mathrm{~N}_{4} \mathrm{SF}$ & 298.34 & $198-200$ & 0.71 & 44.96 \\
\hline TD7 & $\mathrm{C}_{17} \mathrm{H}_{16} \mathrm{~N}_{4} \mathrm{SO}$ & 324.40 & $246-250$ & 0.74 & 38.58 \\
\hline
\end{tabular}

aChloroform: Ethanol (4:0.2)

Table 6: Characterization data for TD1- TD7.

developing diabetic symptoms [18]. Table 7 shows the results of in vitro studies based on pancreatic $a$-amylase inhibition. Only TD7 showed inhibition comparable to the standard drug acarbose, used in the study.

\section{In vivo activity}

In vivo study carried out on alloxan induced diabetes rat model showed that out of all molecules three compounds were found most potent. Glibenclamide which belongs to second generation sulphonylurea class was used as standard at dose $10 \mathrm{mg} / \mathrm{kg}$. Table 8 shows effect of glibenclamide $10 \mathrm{mg} / \mathrm{kg}$ p.o and compounds TD1TD7 (35 mg/kg, p.o.) on blood glucose level by in vivo study of alloxan induced diabetic rat model.

Table 8: In vivo antidiabetic activity of thiadiazole compounds (TD1 -TD7).

Data expressed as mean \pm SEM., and analyzed by Boneferroni post hoc test, using two way ANOVA, $\mathrm{n}=6$ rats per group; $\mathrm{a}$ : $\mathrm{P}<0.001$, as

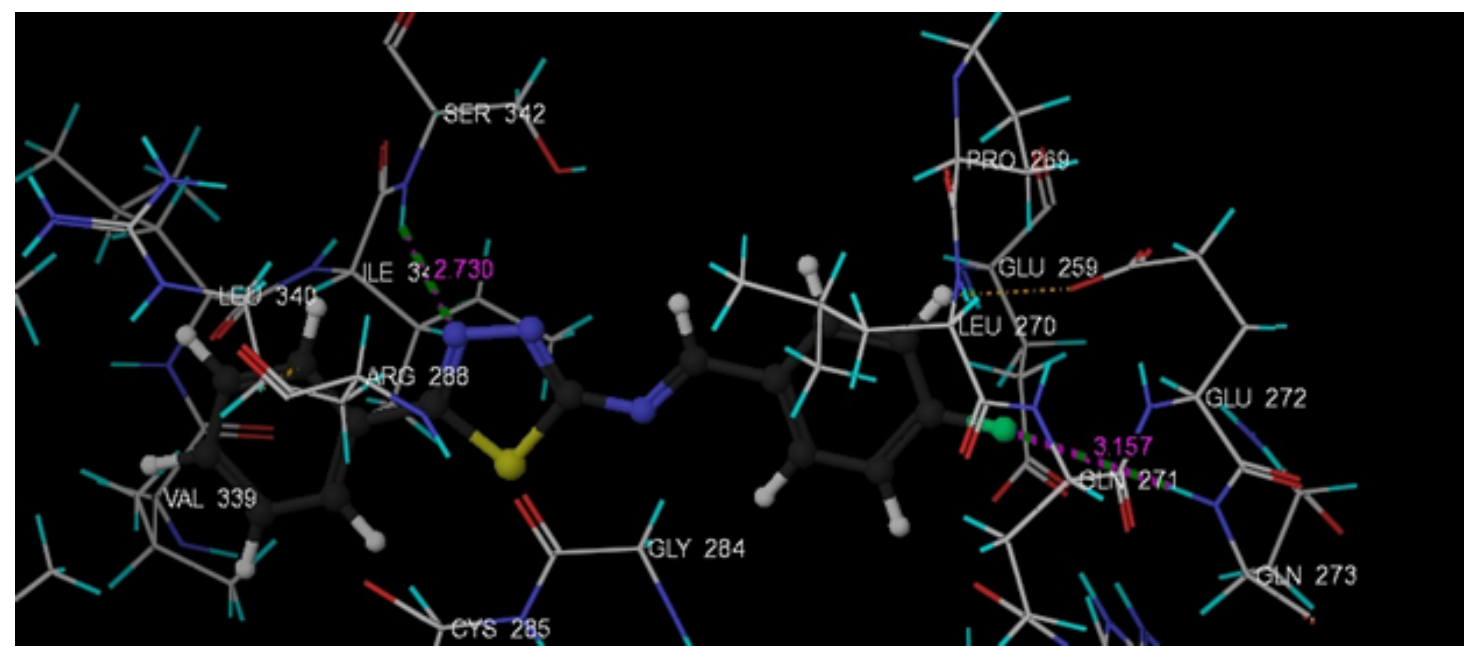

Figure 4: Docking of TD5 showing H-bonding with Ser342 and Glu272. 
Citation: Datar PA, Deokule TA (2014) Design and Synthesis of Thiadiazole Derivatives as Antidiabetic Agents. Med chem 4: $390-399$. doi:10.4172/2161-0444.1000170

\begin{tabular}{|c|c|c|}
\hline Compound & Antidiabetic activity ( $\alpha$-amylase inhibition) & $\mathrm{IC}_{50}$ value $\left(\mu \mathrm{g} \mathrm{ml}^{-1}\right)$ \\
\hline TD1 & $-v e$ & ND \\
\hline TD2 & $-v e$ & ND \\
\hline TD3 & -ve & ND \\
\hline TD4 & $-v e$ & ND \\
\hline TD5 & $-v e$ & ND \\
\hline TD6 & -ve & ND \\
\hline TD7 & $+v e$ & 11.61 \\
\hline Acarbose (Positive Control) & $+v e$ & 10.62 \\
\hline
\end{tabular}

Table 7: Pancreatic $\alpha$-amylase inhibition.

\begin{tabular}{|c|c|c|c|c|}
\hline \multirow{2}{*}{ Compound } & \multicolumn{3}{|c|}{ Blood glucose level $\mathbf{~ m g} / \mathbf{d l}$ (Mean \pm SE) } \\
\hline & $\mathbf{0 ~ h r}$ & $\mathbf{1 ~ h r}$ & $\mathbf{3} \mathbf{~ h r}$ & $\mathbf{6} \mathbf{~ h r}$ \\
\hline Normal & $90.667 \pm 1.585$ & $90.667 \pm 1.585$ & $92.00 \pm 1.438$ & $91.500 \pm 1.996$ \\
\hline TD1 & $207.833 \pm 8.499$ & $129.333 \pm 3.169$ & $118.167 \pm 4.423$ & $11.667 \pm 2.906$ \\
\hline TD2 & $201.00 \pm 4.705$ & $134.833 \pm 2.197$ & $124.833 \pm 4.206$ & $118.00 \pm 3.256$ \\
\hline TD3 & $213.00 \pm 3.406$ & $241.833 \pm 2.762$ & $228.500 \pm 4.507$ & $247.667 \pm 1.600$ \\
\hline TD4 & $204.833 \pm 5.850$ & $209.167 \pm 5.958$ & $214.00 \pm 6.261$ & $214.833 \pm 7.059$ \\
\hline TD5 & $198.00 \pm 3.474$ & $157.833 \pm 3.458$ & $141.667 \pm 3.062$ & $126.833 \pm 2.701$ \\
\hline TD6 & $202.167 \pm 4.729$ & $165.500 \pm 3.888$ & $151.500 \pm 2.029$ & $139.500 \pm 2.884$ \\
\hline TD7 & $200.167 \pm 3.664$ & $158.500 \pm 4.780$ & $140.500 \pm 5.943$ & $106.667 \pm 3.887$ \\
\hline Control & $191.833 \pm 1.701$ & $226.333 \pm 1.338$ & $212.00 \pm 4.494$ & $229.667 \pm 5.942$ \\
\hline $\begin{array}{c}\text { Standard } \\
\text { (Glibenclamide) }\end{array}$ & $201.167 \pm 2.774$ & $116.333 \pm 4.937$ & $113.667 \pm 3.190$ & $109.167 \pm 1.376$ \\
\hline
\end{tabular}

Table 8: In vivo antidiabetic activity of thiadiazole compounds (TD1 -TD7).

compared to diabetic control, @: $\mathrm{P}<0.001$ as compare to normal. The compounds showed comparable antidiabetic activity to the standard drug, glibenclimide used in this study. We hereby statement that all the animal studies have been approved by the institutional ethics committee and have therefore been performed in accordance with the ethical standards laid down in the 1964 Declaration of Helsinki and its later amendments.

In vitro studies shows that compound TD7 showed positive results. From in vivo study, among the compounds synthesized, compounds TD1, TD2, TD7 were found more potent while compounds TD5, TD6 were having moderate activity. Compounds TD3 and TD4 were found less active. The results were calculated by measuring the mean SE \pm ' $\mathrm{P}$ ' value. Hence from both in vitro and in vivo study compound no. TD7 was found potent. All animals survived after the activity. Washing period was given to them so that they could be used for further study.

\section{Conclusion}

From the docking studies seven compounds were selected for synthesis. Antidiabetic activity results showed that out of seven compounds three were found potent and two were found moderate active. Compound TD7 showed significant in vivo antidiabetic activity in alloxan induced diabetes rat model and in vitro pancreatic $a$-amylase inhibition.

\section{Acknowledgement}

We are thankful to Dr. K. G. Bothara for providing the facilities.

\section{References}

1. World Health Organization, Fact sheet, 2013.

2. Kempgowda S, Dev Prakash GP, Tamiz MT (2011) Thiadiazoles: Progress Report on Biological Activities. Der Pharma Chem 3: 330-341.

3. Kamal M, Shakya AK, Talha J (2011) 1, 3, 4-thiadiazoles as antimicrobial agent: A review. Int J Biomed Res 2: 41-61.

4. Singh AK, Mishra G, Jyoti K (2011) Review on Biological activities of 1, 3, 4-thiadiazole derivatives. J Appl Pharm Sci 1: 44-49.

5. Jalhan S, Anil J, Avneet G, Hemraj (2012) Synthesis, biological activities and chemistry of thiadiazole derivatives and Schiff bases. Asian J Pharm and Clinical Res 5: 199-208.

6. Siddiqui N, Ahuja P, Ahsan W, Pandeya SN, Alam MS (2009) Thiadaizoles: Progress report on biologiacal activities. J Chem Pharm Res 1: 19-30.

7. Kalidhar U, Kaur A (2011) 1, 3, 4-thiadiazole derivatives and their biologica activities: A review. Research J Pharm Bio Chem Sci 2: 1091-1106.

8. Hemal B, Dipansu S, Biren S, Dixit CM, Mandev P (2011) Biological profile of thiadiazole. Pharmacologyonline 1: 528-543.

9. Shen L, Zhang Y, Wang A, Sieber-McMaster E, Chen X, et al. (2008) Synthesis and structure-activity relationships of thiadiazole-derivatives as potent and orally active peroxisome proliferator-activated receptors a/d dual agonists. Bioorg \& Med Chem 16: 3321.

10. Mougenot $P$, Namane C, Fett E, Camy F, Dadji-Fa $\tilde{A}^{-}$hun R, et al. (2012) Thiadiazoles as new inhibitors of diacylglycerol acyltransferase type 1. Bioorg Med Chem Lett 22: 2497-2502.

11. Pattan SR, Kekare P, Dighe NS, Nirmal SA, Musmade DS, et al. (2009) Synthesis and biological evaluation of some 1,3,4-thiadiazoles. Journal of Chemical and Pharmaceutical Research 1: 191-198.

12. Pattan SR, Kittur BS, Sastry BS, Jadav SG, Thakur DK, et al. (2011) Synthesis and evaluation of some novel 1,3,4-thiadiazoles for antidiabetic activity. Indian Journal of Chemistry 50B: 615-618.

13. Gianti E, Zauhar RJ (2012) Modeling androgen receptor flexibility: a binding mode hypothesis of CYP17 inhibitors/antiandrogens for prostate cancer therapy. J Chem Inf Model 52: 2670-2683.

14. Arun KP, Nag VL, Panda CS (1999) Studies on the synthesis and bioactivity of some thiadiazole derivatives. Ind J Chem 38B: 998-1001.

15. Ponnusamy S, Ravindran R, Zinjarde S, Bhargava S, Ravi Kumar A (2011) Evaluation of traditional Indian antidiabetic medicinal plants for human pancreatic amylase inhibitory effect in vitro. Evid Based Complement Alternat Med 2011.

16. Fröde TS, Medeiros YS (2008) Animal models to test drugs with potential antidiabetic activity. J Ethnopharmacol 115: 173-183.

17. Van Herck H, Baumans V, Brandt CJ, Boere HA, Hesp AP, et al. (2001) Blood sampling from the retro-orbital plexus, the saphenous vein and the tail vein in rats: comparative effects on selected behavioural and blood variables. Lab Anim 35: 131-139.

18. Bischoff $H$ (1995) The mechanism of alpha-glucosidase inhibition in the management of diabetes. Clin Invest Med 18: 303-311. 